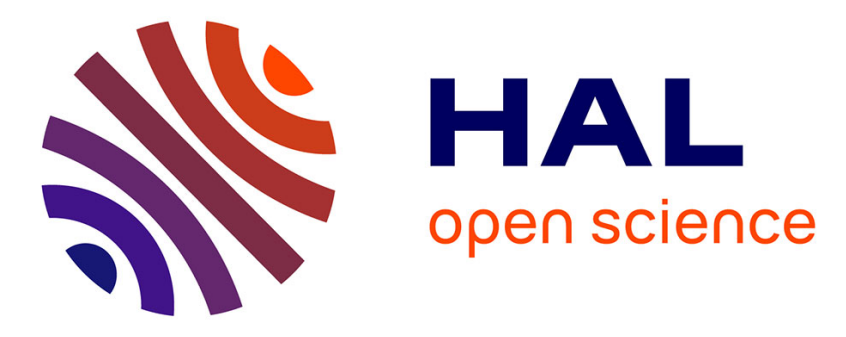

\title{
Dual reciprocity BEM applied to transient elastodynamic problems with differential quadrature method in time
}

\author{
Masataka Tanaka, Wen Chen
}

\section{- To cite this version:}

Masataka Tanaka, Wen Chen. Dual reciprocity BEM applied to transient elastodynamic problems with differential quadrature method in time. Computer Methods in Applied Mechanics and Engineering, 2001, 190 (18-19), pp.2331-2347. 10.1016/S0045-7825(00)00237-1 . hal-01638225

\section{HAL Id: hal-01638225 \\ https://hal.science/hal-01638225}

Submitted on 20 Nov 2017

HAL is a multi-disciplinary open access archive for the deposit and dissemination of scientific research documents, whether they are published or not. The documents may come from teaching and research institutions in France or abroad, or from public or private research centers.
L'archive ouverte pluridisciplinaire HAL, est destinée au dépôt et à la diffusion de documents scientifiques de niveau recherche, publiés ou non, émanant des établissements d'enseignement et de recherche français ou étrangers, des laboratoires publics ou privés. 


\title{
Dual reciprocity BEM applied to transient elastodynamic problems with differential quadrature method in time
}

\author{
Masataka Tanaka*, Wen Chen \\ Department of Mechanical Systems Engineering, Faculty of Engineering, Shinshu University, 500 Wakasato, Nagano 380-8553, Japan
}

\begin{abstract}
This paper is concerned with numerical analysis of the transient elastodynamic problems by the dual reciprocity BEM (DRBEM) in space combined with the differential quadrature method (DQM) in time. Emphasis is placed on a comparative study of various timemarching schemes. Three numerical examples considered are for the longitudinal forced vibration of plates and the transverse free vibration of membranes. To authors' best knowledge, this is the first attempt to apply the DQM to the second-order time derivative in the DRBEM elastodynamic formulations. A recent approach using boundary conditions in the DQM was here extended to handle the initial conditions of elastodynamic problems. The resulting algebraic formulation is a Lyapunov matrix equation, which can be very efficiently solved by the Bartels-Stewart algorithm. It is revealed that the DQM is an unconditionally stable algorithm and gives much better accuracy than the standard finite difference schemes such as the Wilson $\theta$, Newmark and Houbolt methods, for the same time step size for the cases considered.
\end{abstract}

Keywords: Elastodynamics; Dual reciprocity BEM; Differential quadrature method; Time-stepping integration; Lyapunov matrix equation

\section{Introduction}

Recently, the introduction of the dual reciprocity boundary element method (DRBEM) [1-3] has been bringing a major breakthrough in the BEM analysis of time-dependent problems. The DRBEM has been widely recognized as a technique which can retain the boundary-only merit in the BEM analysis of general nonlinear and linear time-dependent problems [3]. Nowadays, the application of the DRBEM to various transient problems has been a subject of growing interest. The boundary integral equations for the DRBEM are dependent only on geometrical data and free of interior cells. The resulting DRBEM formulation of initial-boundary value problems is therefore expressed in the standard form of ordinary differential equations of initial value problems, which can easily be solved by the mature time integrators.

In conjunction with the DRBEM discretization in space, the predominant numerical procedures currently used for time integration in analysis of elastodynamic problems are finite difference approximations such as the Newmark, Houbolt and Wilson methods, which is similar to the situation in the FEM. In compound sources of errors due to approximate representations of spatial and time derivatives, it is seen that the accuracy and computing efficiency of the resulting solutions depend greatly on the proper choice of time-marching schemes.

\footnotetext{
* Corresponding author. Tel.: +81-26-269-5120; fax: +81-26-269-5124.

E-mail addresses: dtanaka@gipwc.shinshu-u.ac.jp (M. Tanaka), chenw@homer.shinshu-u.ac.jp (W. Chen).
} 
Some pioneer works on the DRBEM analysis of elastodynamics are due to Nardini and Brebbia [1] and Loeffler and Mansur [4]. They have concluded that the Houbolt method is preferred in the DRBEM since the artificial damping inherent with this method can effectively depresses influence of higher modes in the response. Kontoni and Beskos [5] also followed this idea to choose the Houbolt method in the DRBEM analysis of dynamic elastoplastic problems. However, this conclusion is not in agreement with the common sense in the FEM dynamics analysis that the Newmark method is normally preferred to other methods [6]. There exist some definite shortcomings in the Houbolt method; it is recognized that high numerical damping in the Houbolt method often impairs the accuracy of the solutions due to undesirable amplitude attenuation if large time step is used, especially for structural dynamic problems in which the response is often dominated by the low-frequency components of the system [7]. Furthermore, Hilber and Hughes [8] have pointed out that the self-starting is one of essentially desirable properties of a competitive numerical integrator for most elastodynamic problems, while the Houbolt method necessarily requires a distinct starting procedure and fails to satisfy this condition. The time integrators of such type not only increase the programming labor but also may cause some complexity of computing. Numerical experiment which preferred to the Houbolt method in $[3,4]$ was an elastic wave propagation problem in which the contribution of high frequency structural modes to the response is important. However, in the cases of such type, the explicit algorithms are very efficient and rather frequently used in practice [7]. In addition, the time step size used in $[3,4]$ was very small, and therefore, those experiments cannot adequately provide an explicit comparison among a variety of integrators. It is clear from the preceding discussions that there is still a strong need to further investigate various numerical integrators in the DRBEM elastodynamic analysis.

On the other hand, Singh and Kalra [9] presented a comprehensive comparative study on the time integrators in the context of the DRBEM formulation of transient diffusion problems involving only the firstorder time derivative. They concluded that the one step least squares algorithm was the most accurate and efficient numerical integrators among all the ones assessed. However, for the problems involving the Dirichlet boundary condition, all time integration methods used in [9] encountered a sharp drop in accuracy and efficiency of computation. Very recently, the present authors [10] employed the differential quadrature method (DQM) to approximate the first-order time derivative in the DRBEM analysis of the same transient diffusion problems investigated in [9]. It was found that the obtained solutions of the Dirichlet problems are very accurate with comparatively much less computing effort than the other existing methods. This work has also revealed that the inefficiency of the DRBEM analysis of the Dirichlet problems reported in [9] are due to the numerical integrator in time rather than the DRBEM discretization in space.

The success in applying the DQM for the diffusion problems has been encouraging the present authors to further extend the method along with the DRBEM to the elastodynamic problems with the second-order time derivatives. This paper also places emphasis on a comprehensive comparative study of various standard time-marching schemes in the context of the DRBEM formulation of elastodynamic problems. The in-plane forced vibration of plates and the transverse free vibration of membranes are taken as numerical examples because their analytical solutions are easily obtainable.

The DQM [11,12] may not be well-known in computational community due to its recent origin. The method can be regarded as the "direct approach" of the traditional collocation method in the sense that the governing equations are analogized in terms of physical variables instead of usually fictitious expansion coefficients. Some details on this method are presented. It has been well known that the DQM as well as the collocation method shows the exponential rate of convergence. The weakness of this method is its flexibility for irregular geometry. Since the time domain has no such difficulty, the strengths of higher-order accuracy of the DQM can be fully exploited in approximating the time derivatives. In addition, the DQM also holds the unconditionally stable feature for accuracy of order more than two. This is due to the fact that the method is not a traditional time step scheme and circumvents the rigorous limitation of accuracy for unconditionally stable algorithms due to Dahlquist theorem [13]. On the other hand, the DQM advances progressively in time domain element-wisely from the initial state, and thus keeps the simplicity and flexibility of the standard time step methods. The resulting set of algebraic equations is in fact the Lyapunov matrix equation and can be very efficiently solved by the well-established Bartels-Stewart algorithm [14]. The total computing effort required in the DQM is comparable to the common implicit step methods. Following the ideas of recent work by Wang and Bert [15] for boundary value problems, this paper also 
develops a simple technique to exactly satisfy all the initial conditions in the DQM analogue of the dynamic systems with the second-order time derivatives.

\section{Vibration of plates and membranes}

The governing differential equation for the longitudinal vibration of plates and the transverse vibration of membranes can be expressed as

$$
\nabla^{2} u(x, t)=\frac{1}{c^{2}} \frac{\partial^{2} u(x, t)}{\partial t^{2}}, \quad x \in \Omega,
$$

subject to the initial conditions:

$$
\begin{aligned}
& u(x, 0)=u_{0}(x), \\
& \dot{u}(x, 0)=v_{0}(x),
\end{aligned}
$$

and the displacement and traction boundary conditions:

$$
\begin{aligned}
& u(x, t)=\bar{u}(x, t), \quad x \subset \Gamma_{u}, \\
& T(x, t)=\bar{T}(x, t), \quad x \subset \Gamma_{T},
\end{aligned}
$$

where $T=\partial u / \partial n$, in which $n$ is the unit outward normal. It is assumed that the domain $\Omega \in R^{2}$ is bounded by a piece-wise smooth boundary $\Gamma=\Gamma_{u}+\Gamma_{T}$.

In this study, the three typical vibration problems of plates and membranes with analytical solutions available in the literature are considered to evaluate the performances of the DQM, Newmark, Houbolt and Wilson methods in conjunction with the DRBEM spatial discretizations. The spatial variable domains of the test problems are square. It is noted that the analytical solutions of the longitudinal vibration of square plates considered are in agreement with those of one-dimensional rod cases [3,23].

\subsection{Vibration of plate subjected to periodic in-plane force}

The initial conditions of this case are assumed as

$$
\begin{aligned}
& u(x, 0)=0, \\
& \dot{u}(x, 0)=0,
\end{aligned}
$$

and the boundary conditions are specified as

$$
\begin{aligned}
& u(x, t)=0, \quad x_{1}=1, \\
& T(x, t)=0, \quad x_{1}=0, \quad x_{2}=0.1 .
\end{aligned}
$$

When a periodic in-plane force

$$
p=P \cos \frac{3 \pi}{8 a} \sqrt{\frac{E}{\rho}} t
$$

is applied at the left side of plate as shown in Fig. 1, we can derive the analytical solution [16,17]:

$$
\left.u=\frac{8}{\pi^{2}} \frac{P a}{E A} \sum_{i=1}^{\infty} \frac{(-1)^{i-1}}{(2 i-1)^{2}-9 / 16}\right) \sin \left(\frac{2 i-1}{2 a} \pi x_{1}\right)\left(\cos \frac{3 \pi}{8 a} \sqrt{\frac{E}{\rho}} t-\cos \frac{(2 i-1) \pi}{2 a} \sqrt{\frac{E}{\rho} t}\right),
$$




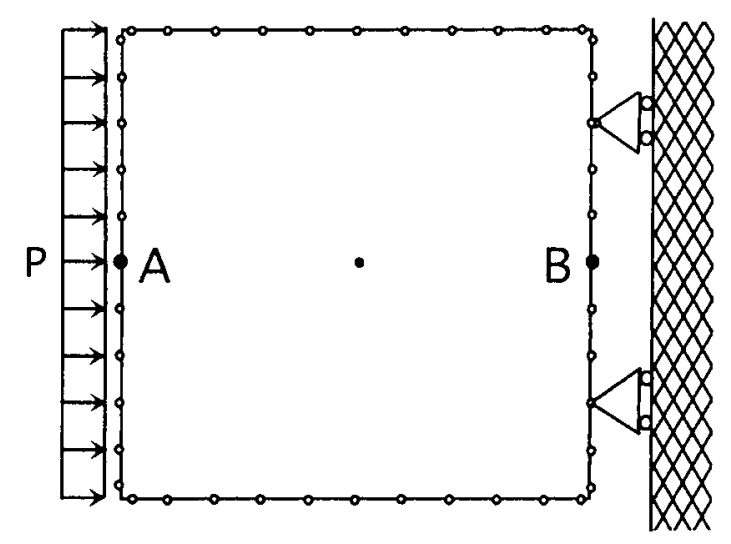

Fig. 1. Linear elements and interior points of a square plate.

where $a$ denotes the length of the plate edges; $A$ is area of cross-section; $E$ is the Young modulus and $p$ the density of the plate material. The traction can be obtained by

$$
T=E A \frac{\partial u}{\partial x}
$$

\subsection{Free vibration of membranes}

It is assumed that a square membrane of $a \times b$ is released from rest in an initial position and velocity, i.e.,

$$
\begin{aligned}
& u(x, 0)=\left(a x_{1}-x_{1}^{2}\right)\left(b x_{2}-x_{2}^{2}\right), \\
& \dot{u}(x, 0)=0 .
\end{aligned}
$$

The boundary conditions are given by

$$
u(x, t)=0 \quad \text { at } x_{1}=-a, a \quad \text { and } \quad x_{2}=-b, b .
$$

By using formulas given in [18], the analytical solution for this case is given by

$$
u(x, t)=\sum_{m, n=1}^{\infty} \frac{16^{2} a^{2} b^{2}}{\pi^{6}} \frac{1-(-1)^{m}}{m^{3}} \frac{1-(-1)^{n}}{n^{3}} \sin \left(\frac{m \pi x_{1}}{2 a}\right) \sin \left(\frac{n \pi x_{2}}{2 b}\right) \cos \left(k_{m n} c t\right),
$$

where

$$
c=\sqrt{\frac{T}{\rho}}, \quad k_{m n}^{2}=\pi^{2}\left(\frac{m^{2}}{4 a^{2}}+\frac{n^{2}}{4 b^{2}}\right),
$$

$T$ and $\rho$ are tension and density, respectively.

\subsection{Vibration of plate subjected to Heaviside-type impact load}

In this case, the initial and boundary conditions are the same as Eqs. (5a)-(6b) of example 1. An impact load of Heaviside-type

$$
p=P, \quad t \geqslant 0
$$


is enforced at the left side of plate as shown in Fig. 1. The analytical solution is given by [17]

$$
\left.u=\frac{8}{\pi^{2}} \frac{P a}{E A} \sum_{i=1}^{\infty} \frac{(-1)^{i-1}}{(2 i-1)^{2}} \sin \frac{(2 i-1) \pi x_{1}}{2 a} 1-\cos \frac{(2 i-1) \pi}{2 a} \sqrt{\frac{E}{\rho} t}\right) .
$$

The traction can be evaluated by Eq. (9).

\section{DRBEM discretization in space}

The governing equation (1) can be weighted by the fundamental solution $u^{*}$ of Laplace operator as follows:

$$
\int_{\Omega}\left(\frac{1}{c^{2}} \frac{\partial^{2} u}{\partial t^{2}}-\nabla^{2} u\right) u^{*} \mathrm{~d} \Omega=0
$$

Applying Green's second identity to Eq. (15) yields

$$
d_{i} u_{i}+\int_{\Gamma}\left(T^{*} u-u^{*} T\right) \mathrm{d} \Gamma=-\int_{\Omega} \frac{1}{c^{2}} \frac{\partial^{2} u}{\partial t^{2}} u^{*} \mathrm{~d} \Omega,
$$

where subscript $i$ denotes the source point, $T^{*}=\partial u^{*} / \partial n$, and $d_{i}=\int_{\Omega} \delta(\zeta, x) \mathrm{d} \Omega$. The dual reciprocity method transforms the domain integral on the right-hand side of Eq. (16) by means of a set of coordinate function $f^{j}(x)$ :

$$
\ddot{u}(x, t) \approx \sum_{j=1}^{N+L} f^{j}(x) \ddot{\alpha}^{j}(t),
$$

where the superimposed dot represents the time derivative, $\alpha^{j}$ are unknown functions of time, and $N$ and $L$ are the numbers of the boundary and selected internal nodes, respectively. The coordinate functions used in this paper were presented by Wrobel and Brebbia [2]. These functions are also linked with $\psi^{j}(x)$ through

$$
\nabla^{2} \psi^{j}=f^{j}
$$

Therefore, we have

$$
\int_{\Omega} \frac{\partial^{2} u}{\partial t^{2}} u^{*} \mathrm{~d} \Omega=\sum_{j=1}^{N+L} \int_{\Omega} u^{*} \nabla^{2} \psi^{j} \mathrm{~d} \Omega
$$

Eq. (15) can finally be reduced to

$$
d_{i} u_{i}+\int_{\Gamma}\left(T^{*} u-u^{*} T\right) \mathrm{d} \Gamma=\sum_{j=1}^{N+L}\left[d_{i} \psi_{i}^{j}+\int_{\Gamma}\left(T^{*} \psi^{j}-u^{*} \eta^{j}\right) \mathrm{d} \Gamma\right] \frac{\ddot{\alpha}}{c^{2}},
$$

where $\eta^{j}=\partial \psi^{j} / \partial n$. Note that $\psi^{j}$ and $f^{j}$ are known functions. The resulting DRBEM formulation for the present transient elastodynamic problems is given by

$$
\boldsymbol{M} \ddot{\boldsymbol{u}}+\boldsymbol{H u}-\boldsymbol{G T}=0,
$$

where $\boldsymbol{M}=(\boldsymbol{G} \boldsymbol{E}-\boldsymbol{H} \Psi) \boldsymbol{F}^{-1}$ is the mass matrix; $\boldsymbol{H}$ and $\boldsymbol{G}$ denote the whole matrices of boundary element with kernels $T^{*}$ and $u^{*}$, respectively. $\boldsymbol{F}, \boldsymbol{\Psi}$ and $\boldsymbol{E}$ are comprised of the coordinate function column vectors $f^{j}$, $\psi^{j}$ and $\eta^{j}$. The discretization procedure in detail can be found in [3].

If displacement boundary conditions are involved in the problems, Eq. (21) is a differential algebraic system. By using an approach of matrix partition $[1,2]$

$$
\boldsymbol{u}=\left\{\begin{array}{l}
u_{1} \\
u_{2}
\end{array}\right\} \quad \text { and } \quad \boldsymbol{T}=\left\{\begin{array}{l}
T_{1} \\
T_{2}
\end{array}\right\}
$$




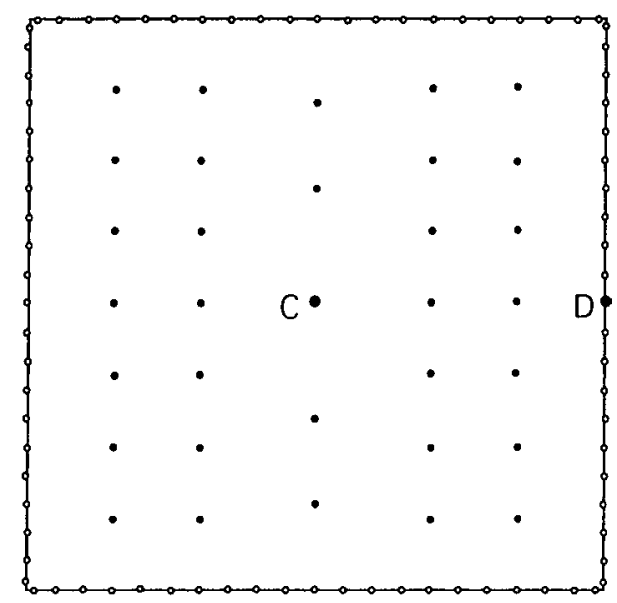

Fig. 2. Linear elements and interior points of a square membrane.

where $\boldsymbol{u}$ and $\boldsymbol{T}$ are divided into two parts corresponding to $\Gamma_{u}$ and $\Gamma_{T}$ parts of the boundary, we can in general have

$$
\hat{\boldsymbol{M}} \ddot{\boldsymbol{u}}_{2}+\hat{\boldsymbol{H}} \boldsymbol{u}_{2}=\overline{\boldsymbol{M}} \boldsymbol{u}_{1}+\overline{\boldsymbol{H}} \boldsymbol{u}_{1}+\overline{\boldsymbol{G}} \boldsymbol{T}_{2}
$$

In particular, we have

$$
\hat{\boldsymbol{M}} \ddot{\boldsymbol{u}}_{2}+\hat{\boldsymbol{H}} \boldsymbol{u}_{2}=\overline{\boldsymbol{G}} \boldsymbol{T}_{2}
$$

for forced vibrations when only external traction is applied, which is the case for examples 1 and 3 in this study. Note that all excitation sources are placed in the right-hand side of Eq. (24). All the coefficient matrices of these equations are dependent only on the geometric data of the problem. The remaining solution procedure is the same as the treatment of the standard initial value problems. The desired traction can be easily calculated after the solutions of the above differential system are accomplished.

The linear element $(\Delta \Gamma=0.1)$ was employed in the present DRBEM discretization as shown Figs. 1 and 2. For examples 1 and 3, one internal point is placed in the center of domain, as shown in Fig. 1. For example 2, 33 internal points are used due to the fact that a homogeneous boundary condition $u=0$ is applied at all the boundary (Fig. 2).

\section{DQM approximation in time}

The DQM is in fact a variant of the standard collocation methods. The advantages of the DQM over the latter were well established in $[11,19]$. First, the practical physical values are directly computed in the DQM, whereas the collocation methods use the indirect expansion (spectral) variables. This greatly simplifies implementations and manifests the DQM in easy-to-choose starting solutions of nonlinear iterations. It is noted that the fictitious expansion variables in the collocation methods usually have not physical meanings and are therefore difficult to choose initial iterative solutions for nonlinear problems [20]. Second, the DQM provides more flexibility to choose grid points to enhance the rate of convergence [19]. For more details of the method see [11] and references cited therein.

The DQM analogue of the first- and second-order derivatives of function $f(t)$ can be expressed as

$$
\left.\frac{\mathrm{d} f(t)}{\mathrm{d} t}\right|_{t_{i}}=\sum_{j=1}^{N} A_{i j} f\left(t_{j}\right),
$$




$$
\left.\frac{\mathrm{d}^{2} f(t)}{\mathrm{d} t^{2}}\right|_{t_{i}}=\sum_{j=1}^{N} B_{i j} f\left(t_{j}\right), \quad i=1,2, \ldots, N,
$$

where $t_{j}$ 's are the discrete points in the temporal variable domain. $f\left(t_{j}\right)$ is the function values at these points, $A_{i j}$ and $B_{i j}$ are the related DQM weighting coefficients for the first- and second-order derivatives, respectively. In the present study, the Chebyshev-Gauss-Lobatto collocation points are used in each time element of the DQM, namely,

$$
t_{i}=\frac{s}{2}\left[1-\cos \left(\frac{i-1}{N-1} \pi\right)\right], \quad i=1,2, \ldots, N,
$$

where $s$ denotes the length of DQM time element and $N$ is the number of grid points.

The DRBEM formulations (23) and (24) can be expressed as the standard form

$$
\ddot{\boldsymbol{u}}+\boldsymbol{K u}=\boldsymbol{f},
$$

where $\boldsymbol{K}=\hat{\boldsymbol{M}}^{-1} \hat{\boldsymbol{H}}$ is stiffness matrix. By using simple algebraic transformation

$$
z=u-u_{0}-v_{0} t+v_{0} t_{0}
$$

where $t_{0}$ is the initial instance of each DQM time element, Eq. (27) is restated as

$$
\ddot{z}+\boldsymbol{K} \boldsymbol{z}=\boldsymbol{F},
$$

where $\boldsymbol{F}=\boldsymbol{f}-\boldsymbol{K} \boldsymbol{u}_{0}-\boldsymbol{K} \boldsymbol{v}_{0} \boldsymbol{t}+\boldsymbol{K} \boldsymbol{v}_{0} \boldsymbol{t}_{0}$, and the respective two initial conditions (2a) and (2b) are set zero, namely,

$$
\begin{aligned}
& \left.z\right|_{t=t_{0}}=0, \\
& \left.\dot{z}\right|_{t=t_{0}}=0 .
\end{aligned}
$$

The above transformation is a key step to apply initial conditions exactly in the DQM approximation of the second-order time derivative. The DQM analogue of the first-order time derivative can be stated as

$$
\bar{A}\{z\}=\{\dot{z}\},
$$

according to the initial condition (30a), and

$$
\bar{A}\{\dot{z}\}=\{\ddot{z}\}
$$

according to the initial condition (30b), where $\overline{\boldsymbol{A}}$ is yielded by removing the first column of the original DQM weighting coefficient matrix $\boldsymbol{A}$ in Eq. (25a). Substituting Eq. (31a) into Eq. (31b), we have

$$
\overline{\boldsymbol{A A}}\{z\}=\bar{B}\{z\}=\{\ddot{z}\},
$$

where the modified DQM coefficient matrix $\bar{B}$ is $(N-1) \times(N-1)$ dimension. It is stressed that the initial conditions specified in Eqs. (30a) and (30b) have been built into the modified coefficient matrix $\overline{\boldsymbol{B}}$. The above scheme is an analogy with the recently developed technique in applying boundary conditions for the DQM solution of high-order boundary value problems presented by Wang and Bert [15].

In terms of approximate formula (32), Eq. (29) can be analogized as

$$
\boldsymbol{Z} \overline{\boldsymbol{B}}^{\mathrm{T}}+\boldsymbol{K} \boldsymbol{Z}=\boldsymbol{F}(\boldsymbol{t})
$$

where $\overline{\boldsymbol{B}}^{\mathrm{T}}$ is a transpose of modified DQM weighting coefficient matrix $\overline{\boldsymbol{B}}$ with the inclusion of two initial conditions given in Eqs. (30a) and (30b). It is noted that $\boldsymbol{Z}$ in Eq. (33) is a rectangular matrix rather than a vector. Therefore, Eq. (33) is a Lyapunov matrix equation. The present DQM procedure discretizes the time variable in the element-wise way, which is somehow different from the standard step-wise solution of single step or multi-step integration. In other words, the present methodology is to advance element by 
element and the multiple grid points are employed in each time element to keep high accuracy of solution. It is worth stressing that the DQM advances progressively in time and thus can maintain the simplicity and flexibility of the common time step methods.

\subsection{Solver of Lyapunov matrix equations}

It is easy to transform the resulting algebraic formulation of the Lyapunov equation (33) into a standard form of simultaneous algebraic equations applicable to being solved by the LU decomposition method. However, such procedure will fail to fully utilize the special structure inherent with the Lyapunov equation so that the computing effort is not necessarily high. In the present study, the so-called Bartels-Stewart algorithm [14] was utilized to solve the Lyapunov algebraic matrix equation (34). The performances of this method are very efficient, stable and accurate. The solution procedures include the following four steps:

Step 1. Reduce $\boldsymbol{K}$ and $\overline{\boldsymbol{B}}^{\mathrm{T}}$ of Eq. (34) into certain simple form via the similarity transformations $\boldsymbol{G}=\boldsymbol{P}^{-1} \boldsymbol{K} \boldsymbol{P}$ and $\boldsymbol{R}=\boldsymbol{V}^{-1} \overline{\boldsymbol{B}}^{\mathrm{T}} \boldsymbol{V}$.

Step 2. $\boldsymbol{Q}=\boldsymbol{P}^{-1} \boldsymbol{F} \boldsymbol{V}$ for the solution of $\boldsymbol{Q}$.

Step 3. Solve the transformed equation $\boldsymbol{G} \boldsymbol{Y}+\boldsymbol{Y} \boldsymbol{R}=\boldsymbol{Q}$ for $\boldsymbol{Y}$.

Step 4. $Z=\boldsymbol{P} \boldsymbol{Y} \boldsymbol{V}^{-1}$.

The time-consuming calculation of $\mathrm{O}\left(M^{3}\right)$ scalar multiplication is required only in Step 1 for Eq. (29) of $M$ dimension, while all implicit step methods also demand $\mathrm{O}\left(M^{3}\right)$ operations using LU decomposition. Moreover, operation in Step 1 need be done only once. On the other hand, Steps 2-4 of the Bartels-Stewart algorithm need be executed repeatedly in each time element, which requires $\mathrm{O}\left(M^{2}\right)$ multiplication. The standard step methods also demand analogous computational effort in each time step. Therefore, the present DQM scheme is comparable in computing effort to that of the normal step implicit methods.

\subsection{A-stability}

It is centrally important whether or not an algorithm is stable in the integration solution of ordinary differential equations. It is known that the collocation method is A-stable [20] which is, in the terminology of computational structural dynamics, unconditionally stable. Therefore, the DQM is also unconditionally stable due to the actual equivalence to the collocation method.

\subsection{Error estimation and accuracy}

The accuracy of the algorithms is of vital importance in computing efficiency and closely related to the truncation error. The error estimator of the DQM approximation of the second-order derivative of function $f(t)$ is given by Chen [12]

$$
\left|R_{i}\right| \leqslant K \cdot \operatorname{err}_{i} \cdot \Delta t^{N-1}, \quad i=1,2, \ldots, N,
$$

where $K=\max \left\{\left|f^{(N)}(\xi)\right|,\left|\xi_{i} f^{(N+1)}(\xi)\right|\right\}$; $\operatorname{err}_{i}$ denotes the error constants dependent on grid spacing and can be obtained easily, $N$ the number of grid points in the DQM time element and $\Delta t$ is time step size.

According to formula (34), the accuracy of the DQM is $\mathrm{O}\left(\Delta t^{N-1}\right)$, for example, ten-order of accuracy when $N=11$. Due to Dahlquist [13], there is no third-order accurate unconditionally stable linear multistep method, and the maximum order of accuracy in the step methods up to two in order to preserve the Astability. The DQM is not a traditional multi-step algorithm and therefore circumvents this rigorous limitation of solution accuracy. The DQM can produce accurate solutions by using larger time step, while still attaining the desirable A-stability merits.

\section{Results and discussion}

In this section, the numerical results of three examples in Section 2 are provided and discussed based a performance comparison of the DQM, Newmark, Houbolt and Wilson methods in conjunction with the 
DRBEM spatial discretization. Parameters $\alpha=0.25$ and $\delta=0.5$ are taken in the Newmark method and $\theta=1.4$ in the Wilson method as in [21]. The coordinates of the displacement, traction and time are dimensionless as $u E / P a$ and $T A / P$ for plate, and $c t$ where $c=1 / a \sqrt{E / \rho}$ for plates and $c=\sqrt{T / \rho}$ for membrane. In this study,

$$
\Delta t=c \frac{s}{N}
$$

where $s$ is the length of the DQM time element, $N$ denotes the number of grid points in the time element and is taken as 11. $\Delta t$ represents the average two-point spacing, which is analogous to the time step size in the usual time step methods.

Figs. 3 and 4 display the solutions of time-displacement curves at point A of Fig. 1 for the longitudinal vibration of square plate subjected to a periodic in-plane force. It is found from Fig. 3 that all methods provide the exact results using sufficiently small time step $\Delta t=0.1$. It is also noted that various finite difference methods confront some small attenuation of amplitude and overshoot for long-term response, while DQ method always gives the very accurate solutions. On the other hand, this also reveals high degree of accuracy of the DRBEM spatial discretization. When a larger time step $\Delta=0.5$ is employed, Fig. 4 illustrates quite distinct performances of various different time-marching schemes. It is observed that the DQM produces strikingly much more accurate solutions than all other time schemes. This demonstrates the superb converging rate and accuracy of the DQM. The Wilson method is found obvious overshoot. In fact, the solutions in long-term response by the various finite difference schemes using the large time step $\Delta t=0.5$ is not acceptable in engineering accuracy for this case.

The time-traction response curves at fixed point B of Fig. 1 for the same example 1 are shown in Figs. 5 and 6. As in the displacement situations, the response by all these methods closely traces the analytical solution curve for the small time step size $\Delta t=0.1$ as shown in Fig. 5. However, as the time step becomes larger $\Delta t=0.5$, the solutions of the traditional finite difference schemes including the Newmark, Houbolt and Wilson methods encounter a sharp drop of accuracy for the long-term response as shown in Fig. 6, while the DQM results always remain in very good agreement with analytical solutions. The Wilson solution is found a strong oscillation at the initial response and evident overshoot in long-term response. Also, all finite difference schemes produce a manifest phase shift.

Figs. 7 and 8 illustrate the analytical and numerical displacement-time curves at central point $\mathrm{C}$ of free vibration of a square membrane. As is expected, all methods produce the exact solutions using the small

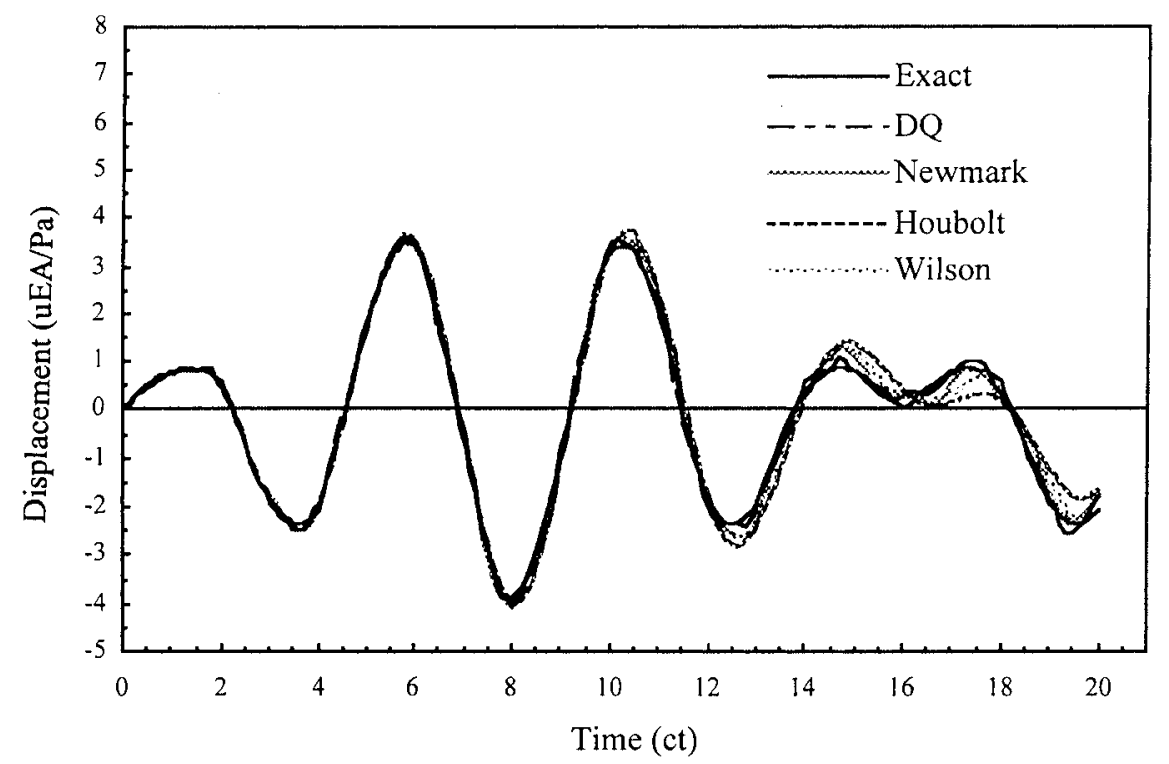

Fig. 3. Displacement curve at point A of a square plate subjected to a periodic in-plane force $(\Delta t=0.1)$. 


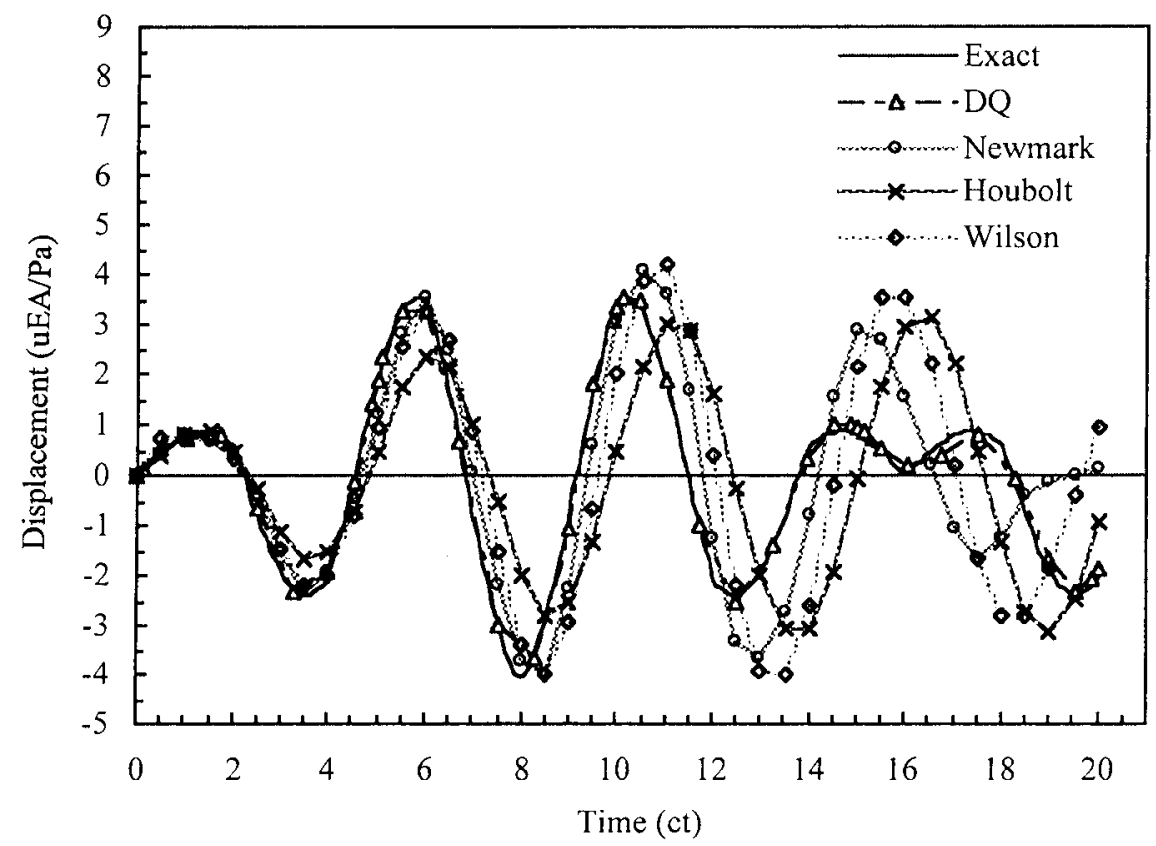

Fig. 4. Displacement curve at point A of a square plate subjected to a periodic in-plane force $(\Delta t=0.5)$.

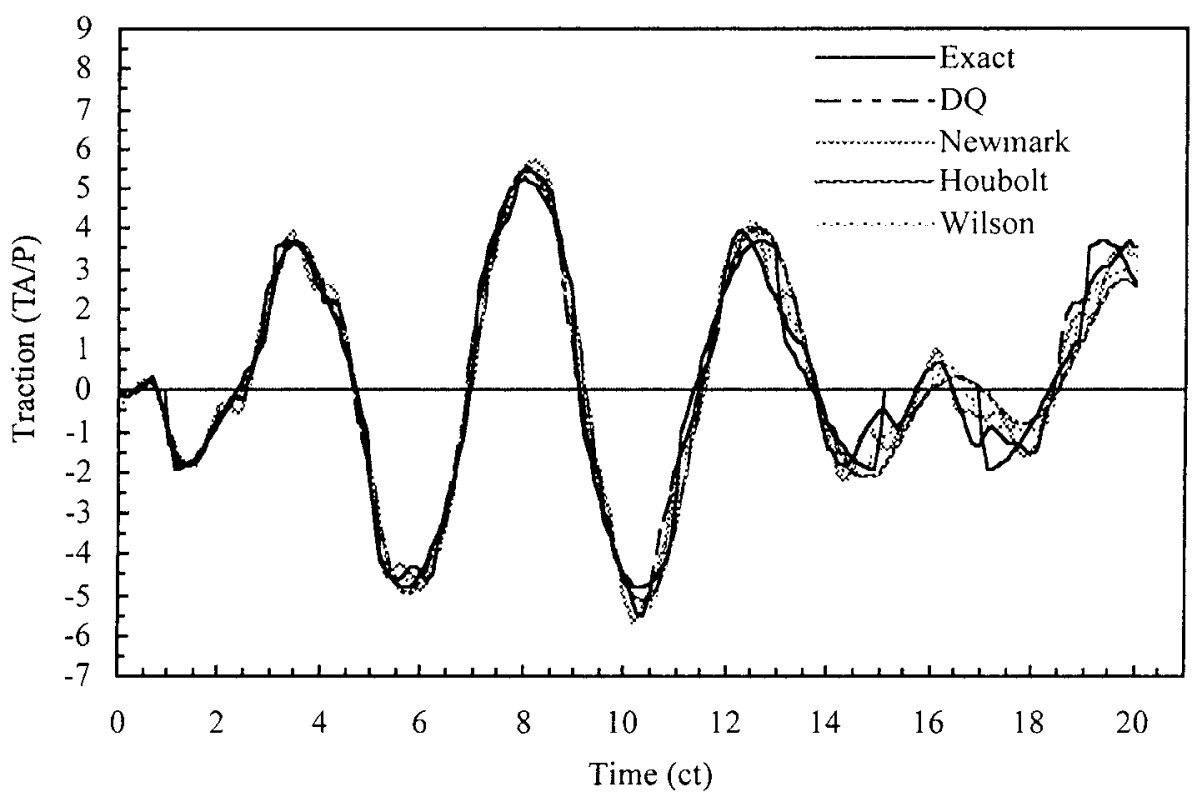

Fig. 5. Traction curve at point $\mathrm{B}$ of a square plate subjected to a periodic in-plane force $(\Delta t=0.1)$.

time step $\Delta t=0.1$ shown in Fig. 7. Among them, the Houbolt solutions have visible phase shift. Significant differences in numerical accuracy, amplitude attenuation, and phase shift are easily observed from Fig. 8 when using larger time step $\Delta t=0.5$. Except for the DQM, all other methods have a great loss of accuracy and evident phase shift. The amplitude attenuation is clearly noted in the Houbolt and Wilson methods, especially in the former due to the excessive artificial damping. To provide more insights into the distinct performances of these methods under coarse time step, Fig. 9 also plotted the normal derivative curves at the boundary point $\mathrm{D}$ in Fig. 2. using $\Delta t=0.5$. Again, the DQM shows the high accuracy, while the 


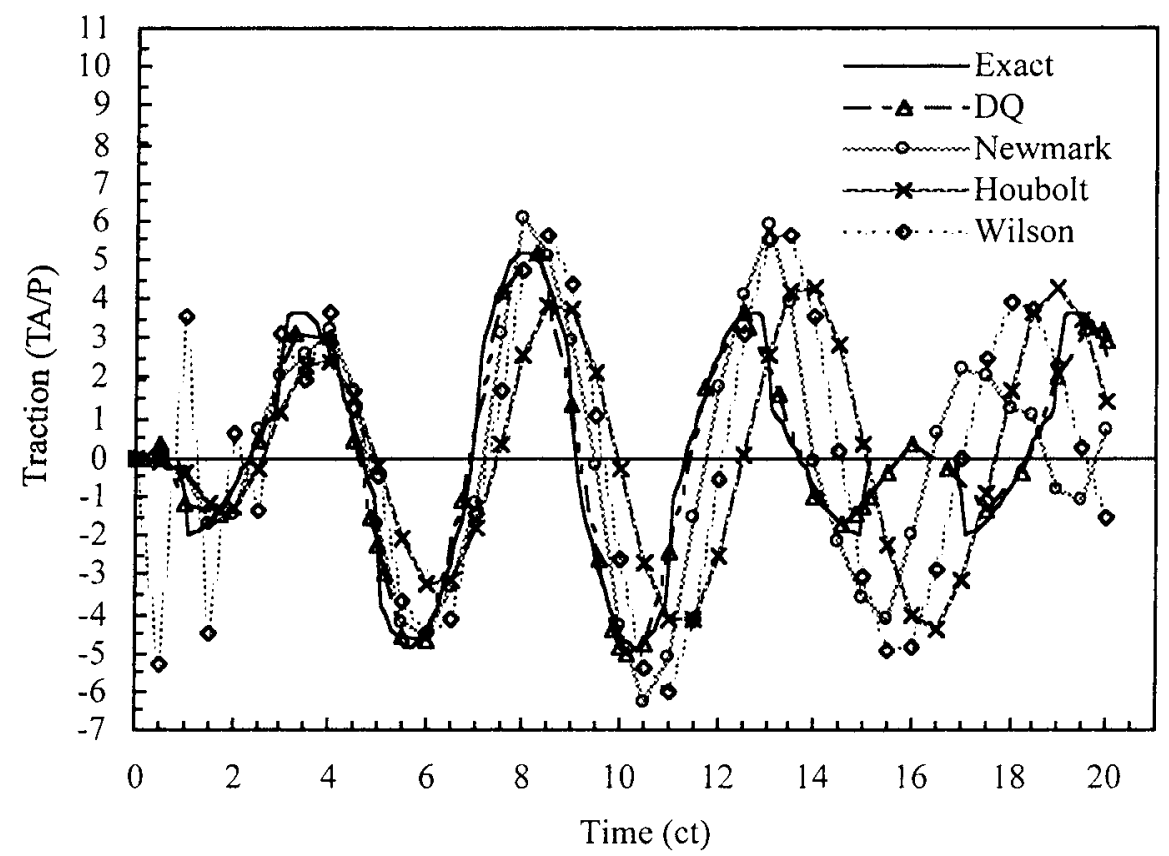

Fig. 6. . Traction curve at point $\mathrm{B}$ of a square plate subjected to a periodic in-plane force $(\Delta t=0.5)$.

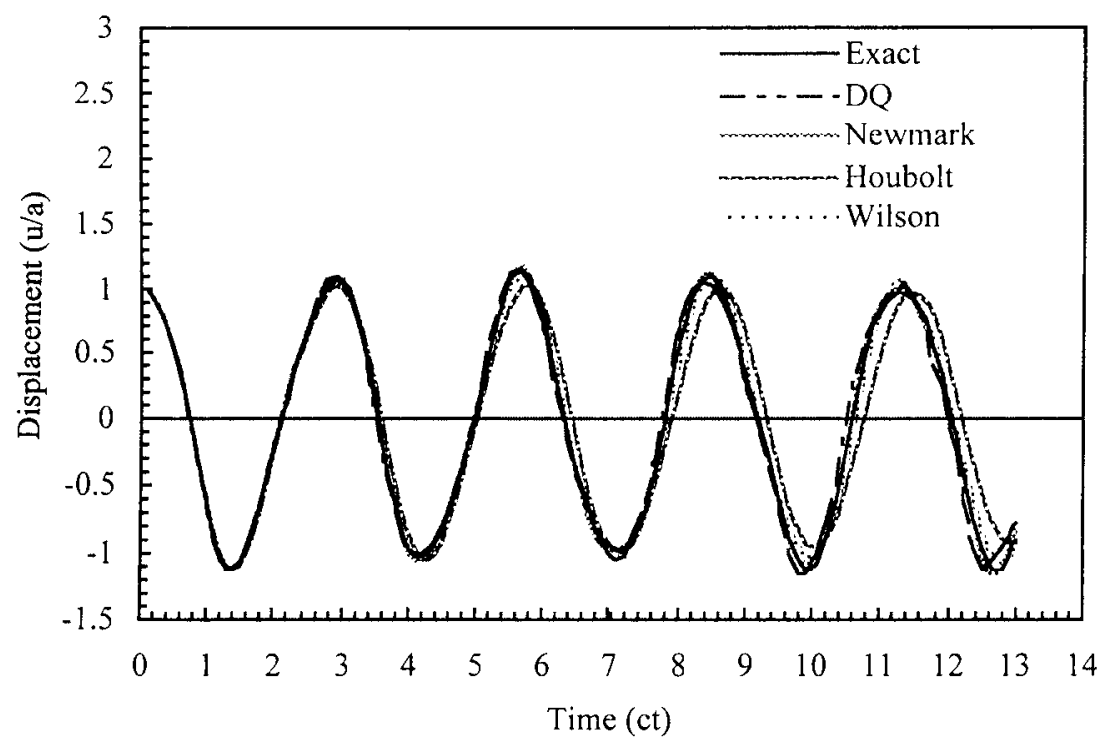

Fig. 7. Displacement curves at center point $\mathrm{C}$ of free vibration of a square membrane with initial displacement $(\Delta t=0.1)$.

Newmark, Houbolt and Wilson methods have a great drop in the solution accuracy and apparent phase shift. In particular, undesirable high numerical damping of the Houbolt method is also observed from Fig. 8.

In conclusion of the above two examples, there are essential differences in the accuracy, amplitude attenuation and phase shift behaviors between the DQM and the standard difference schemes if large time step is chosen for computing economy. This is easily explained by the fact that the DQM is characterized as high accuracy and rate of convergence we referred to din Section 4. It is commonly known that for periodic system analysis, the major factors affecting accuracy of a given method should be truncation error, 


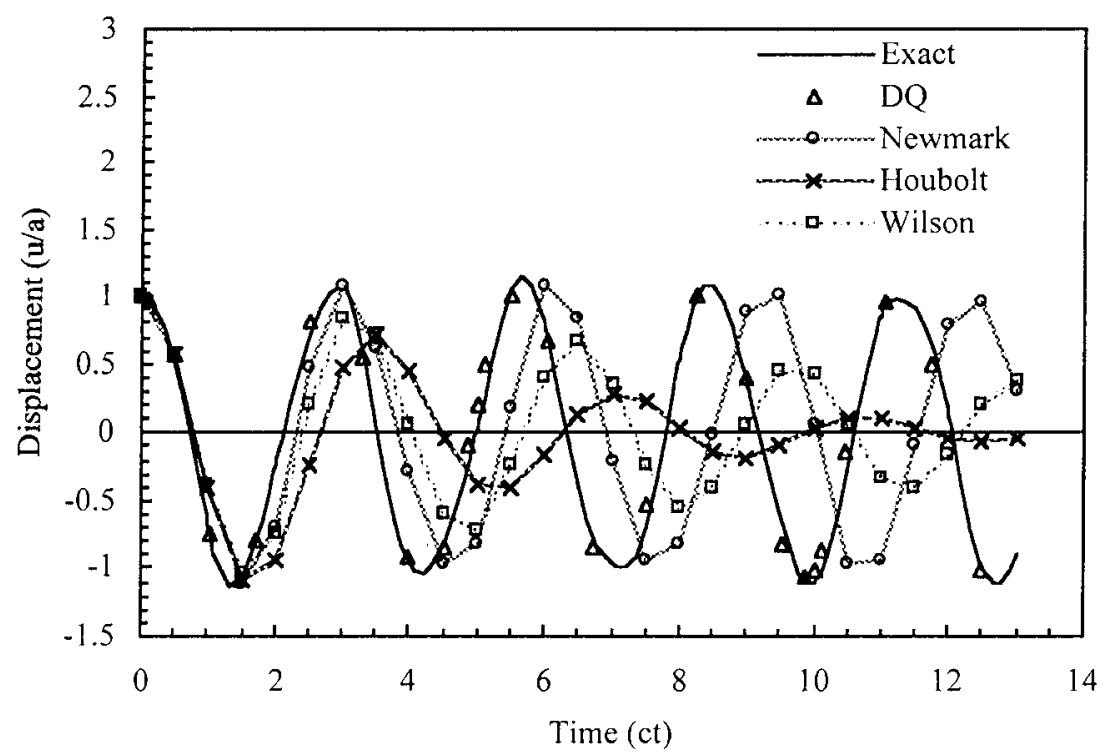

Fig. 8. Displacement curves at center point $\mathrm{C}$ of free vibration of a square membrane with initial displacement $(\Delta t=0.5)$.

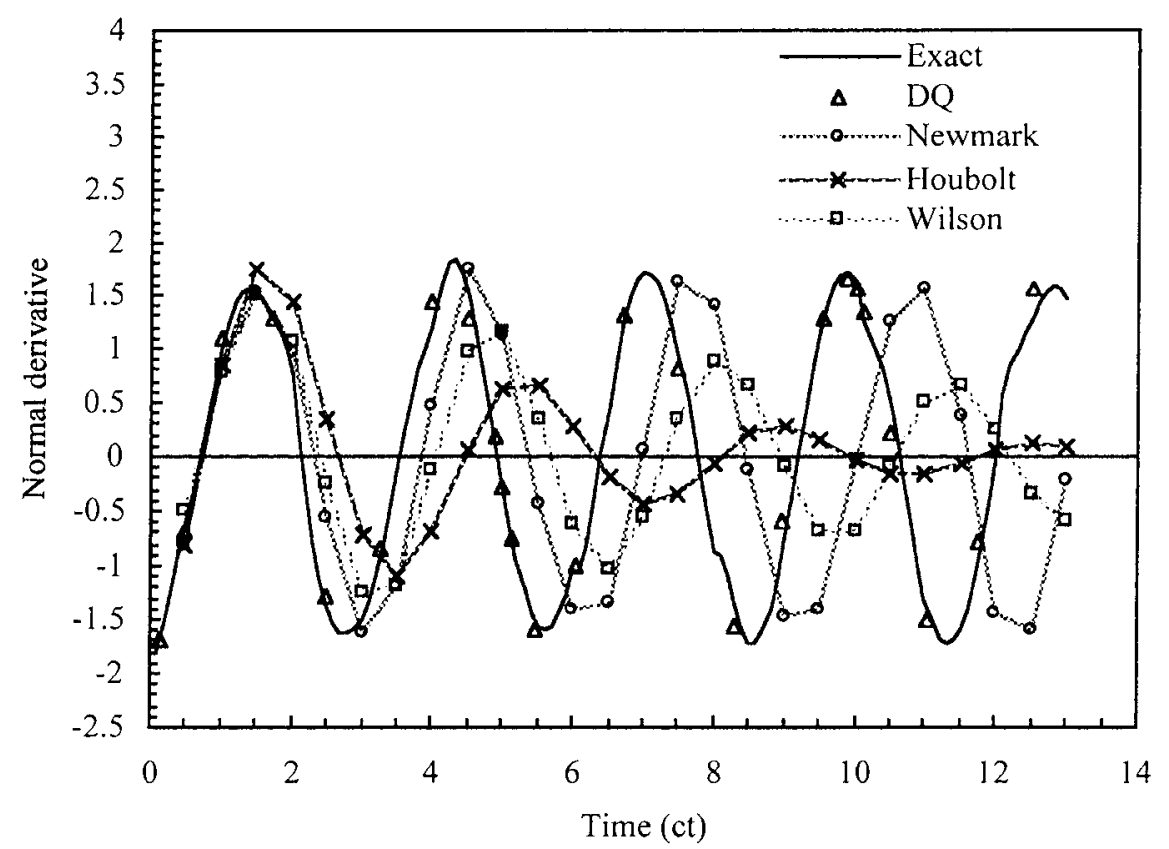

Fig. 9. Normal derivative curve at edge point $\mathrm{D}$ of free vibration of a square membrane with initial displacement $(\Delta t=0.5)$.

numerical damping, and frequency distortion. From the theoretical analysis in Section 4.3 and the given numerical experiments, it is concluded that the DQM retains much higher truncation error and less frequency distortion than the Newmark, Houbolt and Wilson methods.

Example 3 is the longitudinal vibration of a square plate subjected to a Heaviside-type impact. The DQM is tested along with the standard Newmark, Houbolt and Wilson methods. The numerical responses of both displacement at point A and traction at point B are depicted in Figs. 10-14 compared to the corresponding analytical solutions. For small time step $\Delta t=0.1$, it is found from Fig. 10 that all schemes 


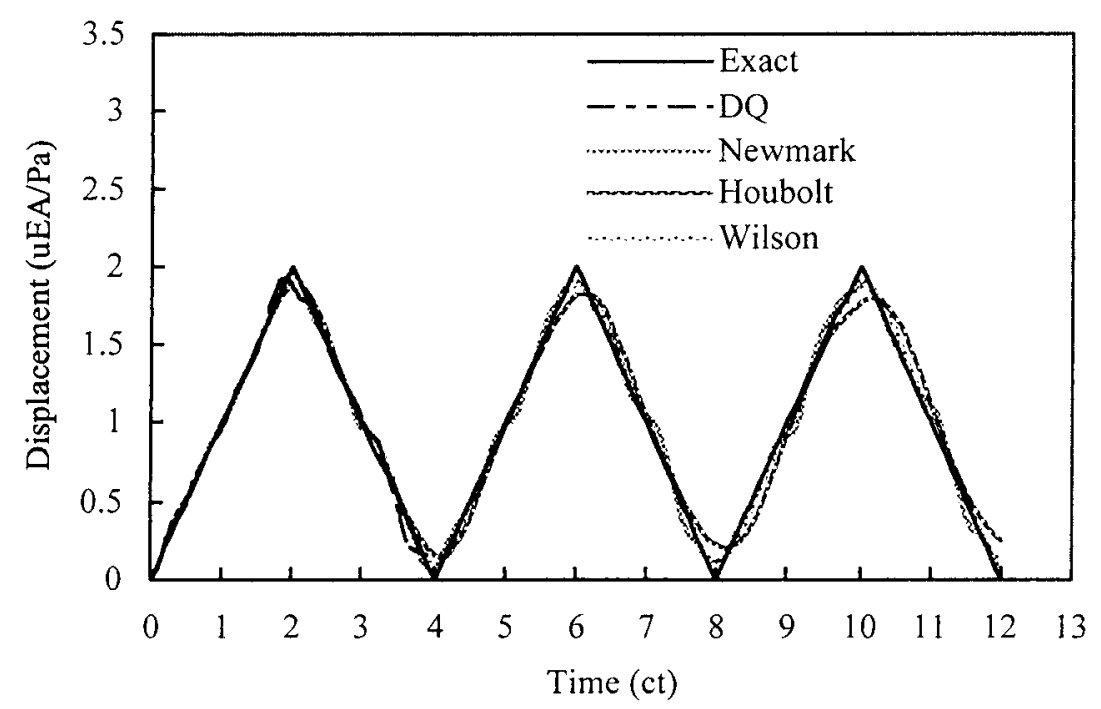

Fig. 10. Displacement curve at point A of a square plate subjected to a Heaviside-type impact $(\Delta t=0.1)$.

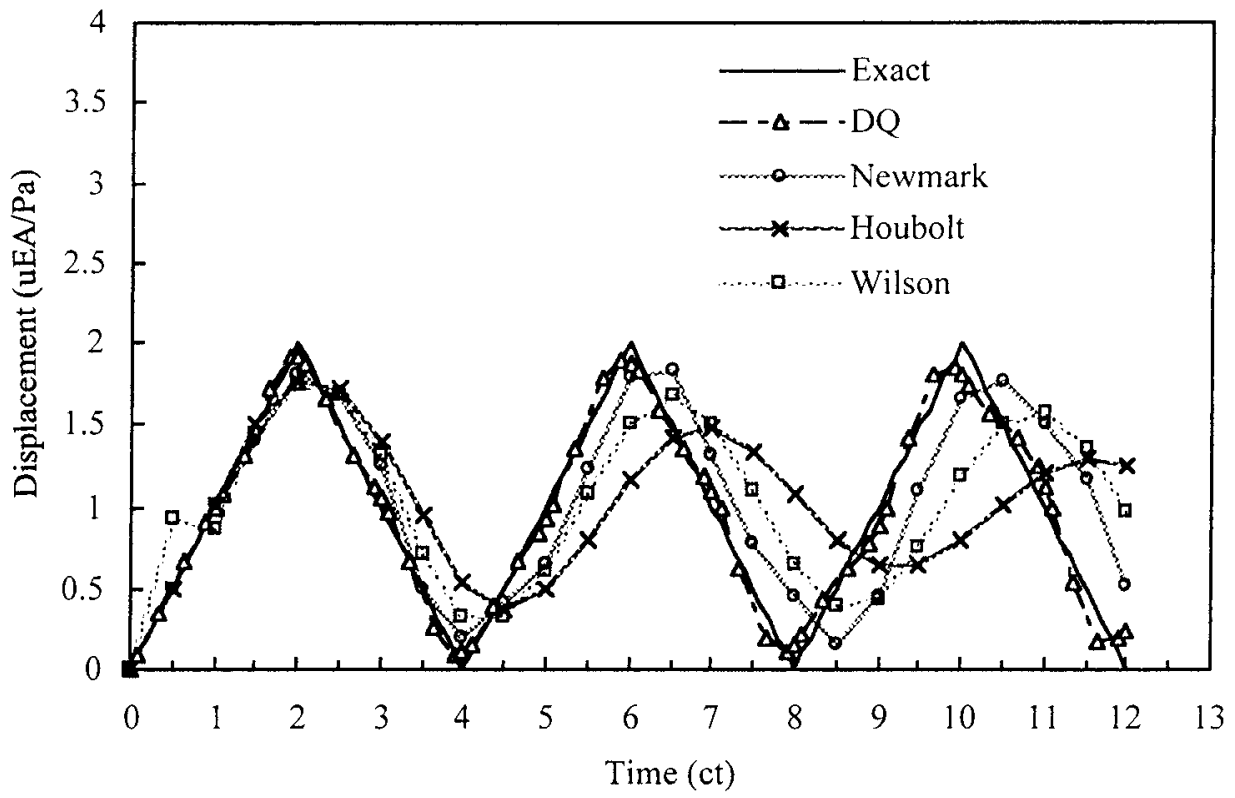

Fig. 11. Displacement curve at point A of a square plate subjected to a Heaviside-type impact $(\Delta t=0.5)$.

can yield the exact solutions of the time-displacement curve. However, as was seen in the previous examples 1 and 2, Fig. 11 shows that the Newmark, Houbolt and Wilson methods confront an obvious fall in solution accuracy when using large $\Delta t=0.5$. Also, an evident amplitude attenuation and phase shift is observed in the Houbolt method. The DQM remains the exact solutions for $\Delta t=0.5$ and behaves very well over any other methods.

High-order modes have a strong effect on the traction response behavior of example 3. This fact is easily observed from Figs. 12-14. It is seen from Fig. 12 that the solutions of all methods have oscillations when very small step size $\Delta t=0.05$ is employed. Among them, the Houbolt method gave the best solutions with the smallest fluctuations. The high artificial damping of the Houbolt method becomes beneficial in this case. This is one of the major reasons in $[3,4]$ to conclude that the Houbolt method is preferred over the other 


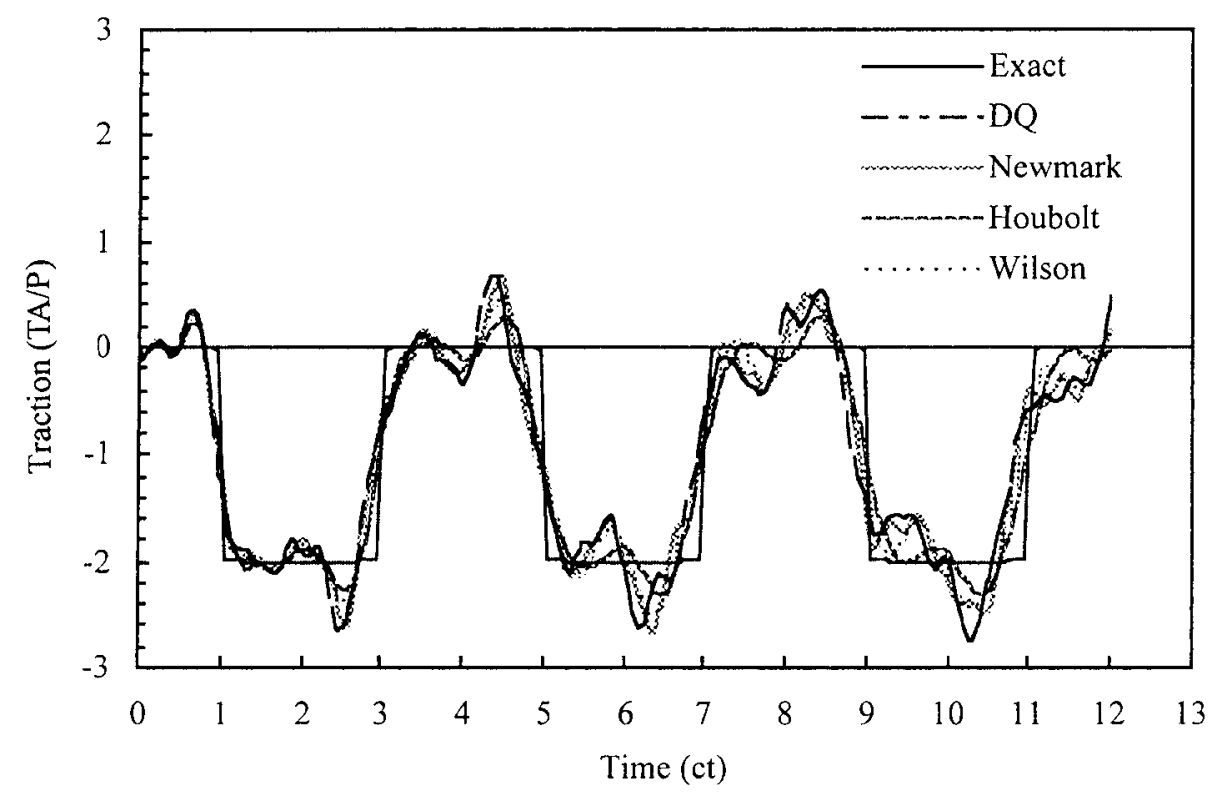

Fig. 12. Traction curve at point $\mathrm{B}$ of a square plate subjected to a Heaviside-type impact $(\Delta t=0.05)$.

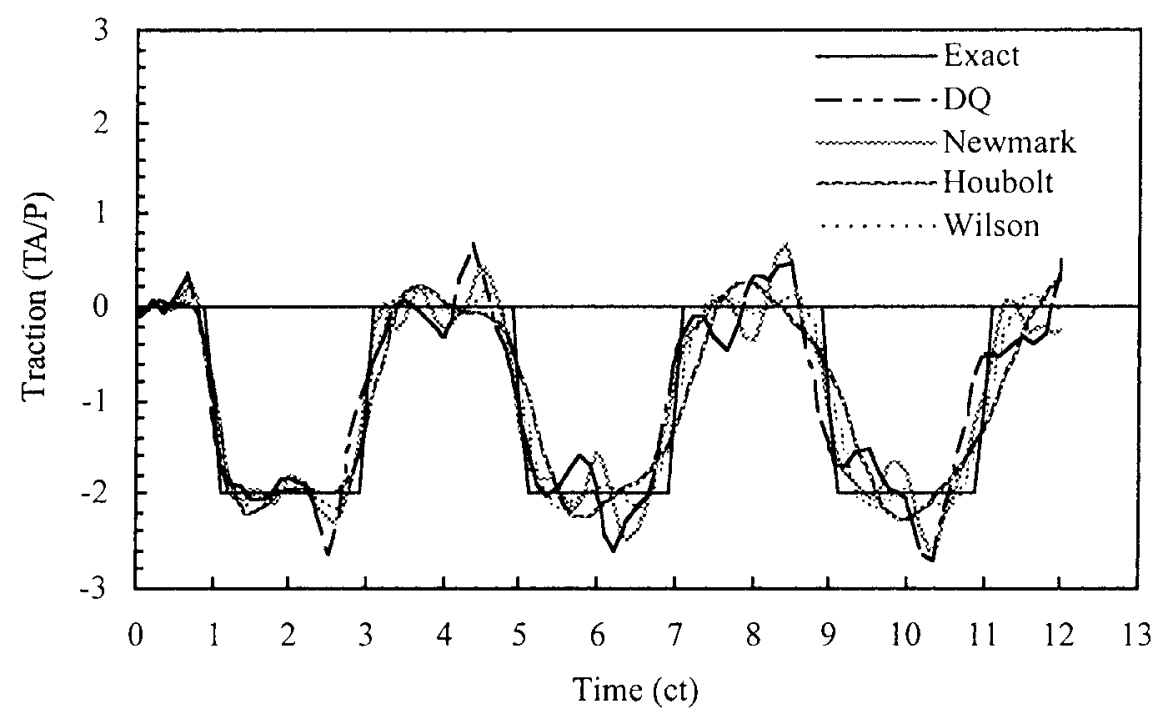

Fig. 13. Traction curve at point $\mathrm{B}$ of a square plate subjected to a Heaviside-type impact $(\Delta t=0.1)$.

finite difference schemes. However, as mentioned previously, it was generally recommended that the explicit method is more efficient for this type of problems in which the high and intermediate frequency components have important affect on the response [7]. The explicit methods are much cheaper and simpler than the implicit algorithms. The weakness in the explicit methods lies in that the numerical stability requires employing the very small time steps. In such case, the explicit methods are much more advantageous in applying the small time step size than the implicit Houbolt method. We confine our attentions in this paper within the implicit methods. For more details on explicit methods see [22].

To investigate the affect of step size on the oscillations of the traction solutions, Figs. 13 and 14 illustrate respectively the traction curves using time step $\Delta t=0.1$ and $\Delta t=0.4$. It is found from Fig. 13 that the 


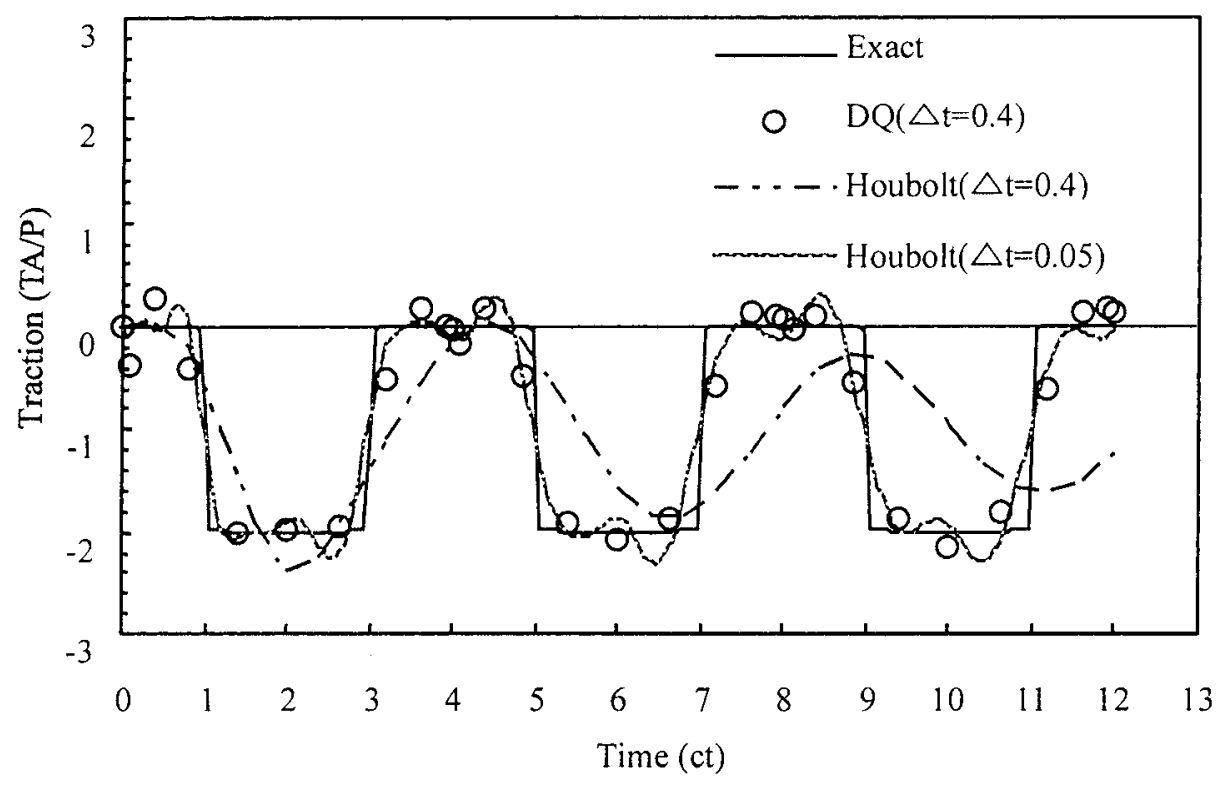

Fig. 14. Traction curve at point $B$ of a square plate subjected to a Heaviside-type impact.

Houbolt method loses some solution accuracy, while the oscillations of the DQM and Newmark method become somewhat weak when using time step $\Delta t=0.1$. The Wilson method emerges comparatively the best time scheme with $\Delta t=0.1$. Furthermore, Fig. 14 depicts the traction curves of the DQM and the Houbolt method. We can find that the oscillation of the DQM solutions using $\Delta t=0.4$ is much less than using $\Delta t=0.05$ in the reference of Fig. 12. Mansur and Brebbia [23] also pointed out the evident relationship between time step size and traction solutions noise of this case, in which a different BEM technique rather than the DRBEM was used to handle domain integral. It is also noted here that the accuracy of the DQM using $\Delta t=0.4$ is almost the same as that of the Houbolt method using $\Delta t=0.05$, while the Houbolt method confronts a great loss of accuracy employing $\Delta t=0.4$. By comparing behaviors of various methods in Figs. 12-14, it is concluded that for the Houbolt method, the time step should necessarily be small enough to produce adequately accurate solutions. In contrast, the DQM method can smooth the solutions and alleviate the oscillation effect of high-order modes by using the larger time step. From the viewpoint of computational economy using coarse time step, the DQM appears the method of choice for this case.

The foregoing discussions indicate that, for the cases where response is primarily dominated by low and intermediate frequency modes, the DQ method exhibits an impressive advantage in the solution accuracy. For the systems on which high modes have important affect such as the traction analysis of example 3 , the DQM is preferred if the large time step is employed and the Houbolt method is the best for the very small time step. However, an explicit algorithm should be considered at first for such type of dynamic systems. It is conceivable that the DQM with other type of basis functions such as harmonic basis functions [24] may yield better characteristics traction analysis of example 3. This is left for further study. Whether the numerical oscillation in computing traction of example 3 is partly due to the spatial discretization also requires further investigation.

\section{Conclusions}

Some important characteristics of numerical integrators for elastodynamics problems have been examined in detail through numerical experiments. It is found that although the Houbolt method seems predominant currently in the solution of the DRBEM formulation of elastodynamics problems [1,3-5], excessive numerical damping in the method can have a very detrimental effect on the accuracy of solutions if large time step is employed, and strengthens the case of applying an alternative method. Based on the 
present study, we conclude that the Newmark method should be in general preferred in the context of the DRBEM formulations of elastodynamics problems in comparison with the Wilson and Houbolt methods.

In this paper, we have applied the DQM to approximate temporal derivative in conjunction with the DRBEM spatial discretization for three typical elastodynamics problems. It is validated through theoretical analysis and numerical experiments that the DQM holds the desirable attributes of unconditional stability yet has higher-order of accuracy than the standard finite difference integrators such as the Newmark, Houbolt and Wilson methods. An effective approach applying the initial conditions was developed in this paper by analogy with a recent work by Wang and Bert [15] in the DQM solution of high-order boundary value problems. The use of the Bartels-Stewart algorithm greatly reduces the computational effort of the DQM to comparable level of the normal implicit finite difference methods. However, the special procedure of the Bartels-Stewart algorithm increases the complexity of the programming. The robustness and superior accuracy of the DQM over the traditional finite difference schemes are clearly observed by comparing the numerical results of three examples. The DQM appears to be a promising technique in practical engineering computations. Further evaluation of this method should be beneficial.

How to satisfy all initial conditions exactly is a key step to successfully implement the DQM to analogize the second-order derivatives in time. There exist two competitive approaches employing multiple boundary conditions in the DQM solution of high-order boundary problems $[11,12,15,25]$. This paper follows the basic idea in [15] to incorporate initial conditions into the modified DQM coefficient matrix. It is still possible to develop a different approach by analogy with the strategy in [12,25]. In addition, the choice of grid spacing in each DQM time element should have some effect on the solution accuracy as in the DQM solution of boundary value problems. The study of the above these problems are now under way.

\section{Acknowledgements}

This work was carried out as a part of the research program supported by the Japan Society for the Promotion of Science. Additional financial support was provided as Grant-in-Aid for JSPS fellows by the Ministry of Education, Science, Sports and Culture, Japan.

\section{References}

[1] D. Nardini, C.A. Brebbia, A new approach to free vibration analysis using boundary elements, Appl. Math. Modelling 7 (1983) $157-162$.

[2] L.C. Wrobel, C.A. Brebbia, The dual reciprocity boundary element formulation for nonlinear diffusion problems, Comput. Methods Appl. Mech. Engrg. 65 (1987) 147-164.

[3] P.W. Partridge, C.A. Brebbia, L.W. Wrobel, The Dual Reciprocity Boundary Element Method, Computational Mechanics Publications, Southampton, 1992.

[4] C.F. Loeffler, W.J. Mansur, Analysis of time integration schemes for boundary element applications to transient wave propagation problems, in: BETECH'87, Computational Mechanics Publications, Southampton, 1987.

[5] D. Kontoni, D. Beskos, Transient dynamic elastoplastic analysis by the dual reciprocity BEM, Engrg. Anal. Boundary Element 12 (1993) $1-16$.

[6] S.J. Kim, J.Y. Cho, W.D. Kim, From the trapezoidal rule to higher-order accurate and unconditionally stable time-integration method for structural dynamics, Comput. Methods Appl. Mech. Engrg. 149 (1997) 73-88.

[7] K. Subbaraj, M. Dokainish, A survey of direct time-integration methods in computational structural dynamics - II. Implicit methods, Comput. Struct. 32 (1989) 1387-1401.

[8] H.M. Hilber, T.J.R. Hughes, Collocation, dissipation and 'overshoot' for time integration schemes in structural dynamics, Earthquake Engrg. Struct. Dynamics 6 (1978) 99-117.

[9] K.M. Singh, S. Kalra, Time integration in the dual reciprocity boundary element analysis of transient diffusion, Engrg. Anal. Boundary Elements 18 (1996) 73-102.

[10] M. Tanaka, W. Chen, Analysis of transient diffusion problems by a combined use of dual reciprocity BEM and differential quadrature method, in: Proceedings of the Fourth conference of the Japan Society for Computational Engineering and Science, Tokyo, 1999, pp. 970-974.

[11] C.W. Bert, M. Malik, Differential quadrature method in computational mechanics: a review, Appl. Mech. Rev. 49 (1996) 1-28.

[12] W. Chen, Differential quadrature method and its applications in engineering - applying special matrix product to nonlinear computations, Ph.D. Thesis, Department of Mechanical Engineering, Shanghai Jiao Tong University, 1996.

[13] G.A. Dahlquist, Special stability problem for linear multistep methods, BIT 3 (1963) 27-43. 
[14] R.H. Bartels, G.W. Stewart, A solution of the equation $A X+X B=C$, Commun. AM 15 (1972) 820-826.

[15] X. Wang, C.W. Bert, A new approach in applying differential quadrature to static and free vibrational analyses of beams and plates, J. Sound Vib. 162 (1993) 566-572.

[16] S. Timoshenko, D.H. Young, W.JR. Weaver, Vibration Problems in Engineering, fourth ed., Wiley, New York, 1974.

[17] K. Kondou, Theory of Vibration, Baihu Kan Press, Tokyo, 1993 (in Japanese).

[18] I. Sneddon, Elements of Partial Differential Equations, McGraw-Hill, New York, 1957 (in Japanese).

[19] J.R. Quan, C.T. Chang, New insights in solving distributed system equations by the quadrature methods - II, Comput. Chem. Engrg. 13 (1989) 1017-1024.

[20] M.K. Burka, Solution of stiff ordinary differential equations by decomposition and orthogonal collocation, AIChE J. 28 (1982) $11-20$.

[21] K. Bathe, E.L. Wilson, Numerical Methods in Finite Element Analysis, Prentice-Hall, Englewood Cliffs, NJ, 1976.

[22] M. Dokainish, K. Subbaraj, A survey of direct time-integration methods in computational structural dynamics - I. Explicit methods, Comput. Struct. 32 (1989) 1371-1386.

[23] W.J. Mansur, C.A. Brebbia, Further development on the solution of the transient scalar wave equation, in: C.A. Brebbia (Ed.), Topics in Boundary Element Research, vol. 2, Springer, Berlin, 1985, pp. 87-123.

[24] A.G. Strize, X. Wang, C.W. Bert, Harmonic differential method and applications to structural components, Acta Mechanica 111 (1995) 85-94.

[25] C. Shu, H. Du, A generalized approach for implementing general boundary conditions in the GDQ free vibration analysis of plates, Int. J. Solids Struct. 34 (1997) 837-846. 